\title{
Synthesis of Ultra Pure Long Normal Alkanes to Hexacohectane, Their Crystallization and Thermal Behavior
}

\author{
Yoshiko Urabe, ${ }^{\dagger}$ Satoshi Tanaka, Seiji Tsuru, Mitsuyuki Fujinaga, Hiroko Yamamoto, \\ and Kanichiro TAKAMIZAWA*
}

Department of Applied Physics, Faculty of Engineering, Kyushu University, Hakozaki, Higashi-ku, Fukuoka 812, Japan

(Received December 18, 1996)

\begin{abstract}
Ultra pure long chain normal alkanes (abbr.: $\mathrm{C} n$, where $n$ is the number of carbon atoms in a chain ), C80, $\mathrm{C} 102, \mathrm{C} 120, \mathrm{C} 160$, were synthesized by Wurtz condensation. For samples of $\mathrm{C} 120$ and $\mathrm{C} 160$, a once-folded chain crystal was formed by solution crystallization; thicknesses of the layer lamella were on an average $7.8 \mathrm{~nm}$ for C120, and $10.0 \mathrm{~nm}$ for C160, which equals to a half of the extended chain length for each molecular weight. In the DSC thermograms of the C160 crystals, an exothermic peak which indicates recrystallization to extended chain crystal following to melting of once-folded chain crystal, appeared clearly. Consequently, folding of normal alkane molecular chain was found to take place from about $\mathrm{C} 120$.

KEY WORDS Higher Normal Alkane / Homologous Purity / Gel Permeation Chromatography / Wurtz Condensation / Once-Folded Chain Crystal / Extended Chain Crystal / Hexacohectane / Eicosahectane
\end{abstract}

One remarkable characteristic of flexible polymers is that they crystallize by chain folding. Since the first report on chain folding in solution crystallization of polyethylene $(\mathrm{PE})^{1} 40$ years ago, many studies have been made on crystallization behavior and resulting crystal structures using well-characterized PE and also normal alkanes (abbr., $n$-alkanes) as model compounds. To elucidate the characteristics of the chain folding phenomenon ultra long $n$-alkanes such as C384 and C390 were synthesized by Wegner et al. $^{2}$ and Whiting et al., ${ }^{3}$ respectively 1985. Unfortunetely they could not give a definite answer enough yet, owing to limited amounts of the synthesized products and insufficient homologous purity.

Takamizawa et $a .^{4}$ sought solution to the same problem by stundying higher-order crystalline structure on low molecular PE fractions $\left(M_{w} / M_{n}=1.01-1.03\right)$ located in the transition region from extended chain crystal to folded chain crystal. The relationship between average molecular weight and X-ray long period $L$ for PE single crystals is in Figure 1. Dotted lines show lamella thickness corresponding to the number of folding, $f=0$, 1 , and 2, calculated from chain length. In this calculation, contribution of the folded portions to the thickness is neglected. At the low molecular weight end, the long period linearly increases, regardless of the crystallization solvent, with the chain length, and $L$ is nearly in accordance with the calculation shown as dotted lines. However, $L$ suddenly decreases when the molecular weight exceeds a critical value depending on the crystallization solvent; i.e., the effects of chain folding are clearly recognized. As a whole, PE single crystals appear to have higher-order structures with integer numbers of folding following the calculated line except single crystals from fractions of $2000-2400$. Takamizawa ${ }^{4}$ printed out that this discrepancy was attributable to polydispersity of samples.

It is very difficult to get a fraction with very narrow

\footnotetext{
$\uparrow$ To whom correspondence should be addressed.

* Sadly passed away. Will be greatly missed.
}

distribution of molecular weight from simple fractionation. Thus, we synthesized ultra pure $n$-alkanes with chain length as long as possible as the model compound of PE. First, $n$-alkanes were synthesized by the Wurtz condensation of polymethylene-dihalide following a report of Carothes et al. ${ }^{5}$ Owing to insufficient purity of the carboxylic acid used as starting material, report ${ }^{6}$ was made only on the pressure dependence of the melting point of $n$-alkanes.

This paper reports the synthesis of high purity $n$ alkanes of $\mathrm{C} 80$ and $\mathrm{C} 102$ whose chain lengthes are in the region corresponding to the lamella thickness of folded chain crystal of PE as well as C120 ${ }^{4}$ and C160 where chain folding starts. The melting behavior of the prepared crystals is discussed based on results concerning the molphology, as well as higher-order structure of folded chain crystals.

\section{EXPERIMENTAL}

\section{Materials}

For sample synthesis, purified linear carboxylic acid and dicarboxylic acid free of homologue were used as starting materials with consideration to elongation of

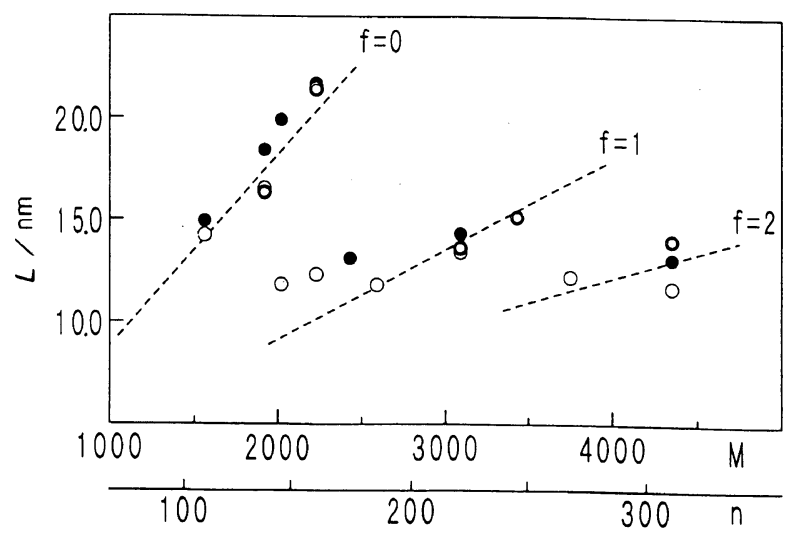

Figure 1. Correlation of long periods with molecular weights of polyethylene fractions. Crystallization solvents: $\bigcirc, p$-xylene (PX); $\mathbf{O}$, iso-amylacetate (IAAC); (), hexadecane. 


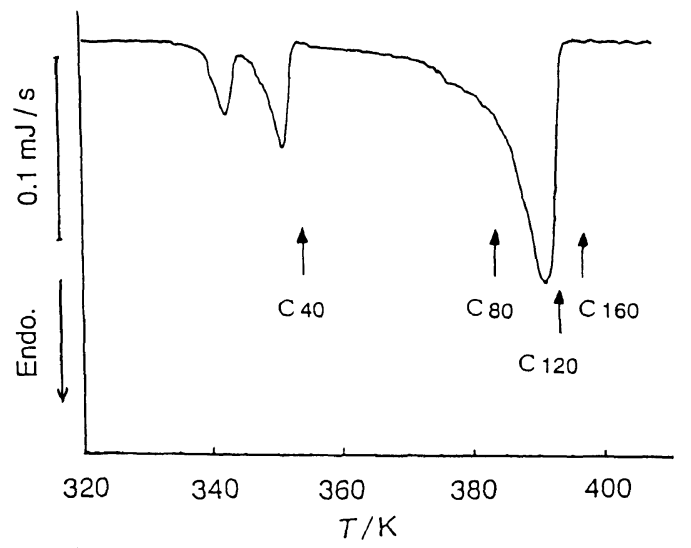

Figure 2. DSC thermograms for synthetic products by Wurtz condensation.

length by the Wurtz condensation ${ }^{5}$ of iodides. Icosanedioic acid (Okamura Seiyu Co.), as the starting material was purified as methyl ester by reperted molecular distillation and recrystallization. Homologous purity was determined by a capillary gas chromatograph (GC) as $99.9 \%$ or higher.

The reaction scheme is as follows.

$$
\begin{aligned}
& \mathrm{HOOC}\left(\mathrm{CH}_{2}\right)_{18} \mathrm{COOH} \rightarrow \mathrm{HO}\left(\mathrm{CH}_{2}\right)_{20} \mathrm{OH} \rightarrow \mathrm{Br}\left(\mathrm{CH}_{2}\right)_{20} \mathrm{Br} \\
& \rightarrow \mathrm{ArO}\left(\mathrm{CH}_{2}\right)_{20} \mathrm{Br} \rightarrow \mathrm{ArO}\left(\mathrm{CH}_{2}\right)_{20} \mathrm{I}(1) \\
&\left(\mathrm{ArO}-=\mathrm{CH}_{3} \mathrm{O}->\mathrm{O}-\right) \\
& \mathrm{H}\left(\mathrm{CH}_{2}\right)_{n-1} \mathrm{COOH} \rightarrow \mathrm{H}\left(\mathrm{CH}_{2}\right)_{n} \mathrm{OH} \rightarrow \mathrm{H}\left(\mathrm{CH}_{2}\right)_{n} \mathrm{I} \text { (2) } \\
&(1)+(2) \rightarrow \mathrm{H}\left(\mathrm{CH}_{2}\right)_{n+20} \mathrm{OAr} \rightarrow \mathrm{H}\left(\mathrm{CH}_{2}\right)_{n+20} \mathrm{I} \\
& \rightarrow \mathrm{H}\left(\mathrm{CH}_{2}\right)_{2 n+40} \mathrm{H}(n=20 ; \mathrm{C} 80, n=31 ; \mathrm{Cl}(n))
\end{aligned}
$$

This is a coupling reaction of 1-iodo-20 ( $p$-methoxyphenoxy)icosane (1) and iodo-alkane (2). Since difference of adsorption is large between the by-products (ArO- $\left.\left(\mathrm{CH}_{2}\right)_{40} \mathrm{OAr}, \mathrm{H}\left(\mathrm{CH}_{2}\right)_{40} \mathrm{H}\right)$ and unreacted materials, separation is possible by a column method. In this way, C80 and C102 were obtained with high purity.

For synthesis of $\mathrm{C} 120$ and $\mathrm{C} 160$, the iodide of the by-product $\operatorname{ArO}\left(\mathrm{CH}_{2}\right)_{40} \mathrm{OAr}, 1,40$-diiodotetracontane was subjected to the Wurtz condensation for chain extension.

$$
\mathrm{I}\left(\mathrm{CH}_{2}\right)_{40} \mathrm{I} \rightarrow \mathrm{H}\left\{\left(\mathrm{CH}_{2}\right)_{40}\right\}_{m} \mathrm{H} \quad(m=1,2,3,4)
$$

Figure 2 shows DSC thermograms of the alkanes prepared. The arrows indicate melting points of the corresponding $n$-alkanes. The mixture was refined by an adsorption column and GPC to get C120 and C160. Separation in GPC was performed at column temperature higher than $90^{\circ} \mathrm{C}$ using toluene or xylene as the elution solvent, and the $0.1 \%$ solution was isolated.

Homologous purity was analyzed by a Shimadzu GC14A capillary GC equipped with a column of SGE 25A Q5-HT-5-0.15 and data processor C-R6A. Carefully programmed temperature operation on the capillary GC ensured that an upper limit of the number of carbon atoms for $n$-alkanes to be analyzed by the capillary GC

\begin{tabular}{|c|c|c|c|c|}
\hline \multirow{2}{*}{$\mathrm{Cn}$} & $L$ & $L_{\text {cal }}$ & $T_{\mathrm{m}}$ & $T_{\text {meal }}$ \\
\hline & $\mathrm{nm}$ & $\mathrm{nm}$ & K & K \\
\hline C80 & 10.6 & 10.5 & 382.8 & 382.8 \\
\hline C102 & 13.1 & 13.3 & 388.3 & 389.0 \\
\hline C120 & $7.8^{*}$ & & & \\
\hline \multirow{3}{*}{ C160 } & $\left(15.0^{*}\right)$ & 15.6 & (392.4) & 392.7 \\
\hline & $10.0^{*}$ & & & \\
\hline & $\left(20.2^{*}\right)$ & 20.7 & (397.5) & 398.1 \\
\hline
\end{tabular}
islocated at around $110 .{ }^{8}$ The samples used in this work was found to have the following purities; $\mathrm{C} 80,99.4 \%$; $\mathrm{C} 102,99.1 \%$; $\mathrm{C} 120$ and $\mathrm{C} 160$, unanalyzable, while $\mathrm{I}\left(\mathrm{CH}_{2}\right)_{40} \mathrm{I}$ before the condensation was of $98.9 \%$ purity.
Table I. Long periods and melting temperatures of ultra long $n$-alkanes ${ }^{\mathrm{a}}$

${ }^{\text {a }} L_{\text {cal }}, T_{\text {mcal }}$ : the calculated from Broadhurst's equation. "Determined from shadow length on TEM photographs ( ) determined from IAAC solution ( $\left.T_{\mathrm{c}} 365 \mathrm{~K}\right)$.

\section{Measurements}

DSC measurements were performed by a Rigaku DSC-8240B with a TAS-100 controller. The standard scanning rate of $0.5 \mathrm{~K} \mathrm{~min}^{-1}$ and sample mass of about $1.00 \mathrm{mg}$ were used. The temperature and heat of transition were calibrated as reported previously. ${ }^{7}$

The X-ray long period $L$ for layered mat of the solution crystal was measured with a Rigaku Geigerflex 2027 diffractometer using $\mathrm{Ni}$ filtered $\mathrm{Cu}-K_{\alpha}$ radiation.

TEM and SEM were made for observation of morphology with a Hitachi MS-5 and Nihondenshi JSMT100, respectively.

\section{RESULTS AND DISCUSSION}

\section{$X$-Ray Long Period and Melting Point of n-Alkanes}

Table I gives values of X-ray long periods $L$ and melting temperatures $\left(T_{\mathrm{m}}\right)$ of $\mathrm{C} 80$ crystal and $\mathrm{C} 102$ crystal prepared from $0.1 \%$ heptane solution by slow cooling method. Agreement of $T_{\mathrm{m}}$ values with those calculated from Broadhurst's equation ${ }^{9}$ indicates that the molecular chains were crystallized as the extended form. Figure 3(a) and (b) show TEM photographs of $\mathrm{C} 160$ and $\mathrm{C} 120$ crystals from $0.05 \%$ p-xylene (PX) solution (a) and the crystals from $0.05 \%$ isoamyl acetate (IAAC) solution (b) at $365 \mathrm{~K}$. The crystals from PX solution were lozenge shaped, and their thicknesses determined from shadow length measurements of TEM photographs were with average $10.0 \mathrm{~nm}$ for $\mathrm{C} 160$ and $7.8 \mathrm{~nm}$ for $\mathrm{C} 120$ crystals, or half the extended chain length for each molecular weight. Long periods of C160 crystal and C120 crystal could not be measured due to insufficient sample amount, but formation of once-folded chain crystal was confirmed. The crystals of $\mathrm{C} 160$ and C120 from IAAC were like tree leaves, and similar to the extended chain crystals which Takamizawa et al ${ }^{10}$ obtained previously from low molecular weight PE fractions. Toda ${ }^{11}$ and Frank ${ }^{12}$ reported that roundshaped PE crystals were obtaind from solution crystallized at high temperature, and discussed the mechanism of crystallization. The values marked by $*$ in Table I were obtained from the shadow length measurement of TEM photographs. Crystal thickness was on an average $20.0 \mathrm{~nm}$ for $\mathrm{C} 160$ and $15.0 \mathrm{~nm}$ for C120 crystals, corresponding to molecular chain length. This means the growth of extended chain crystals in IAAC. 
a)

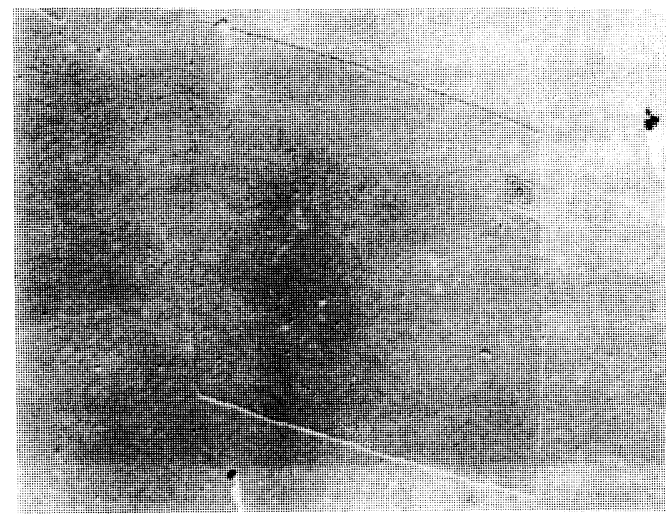

c)

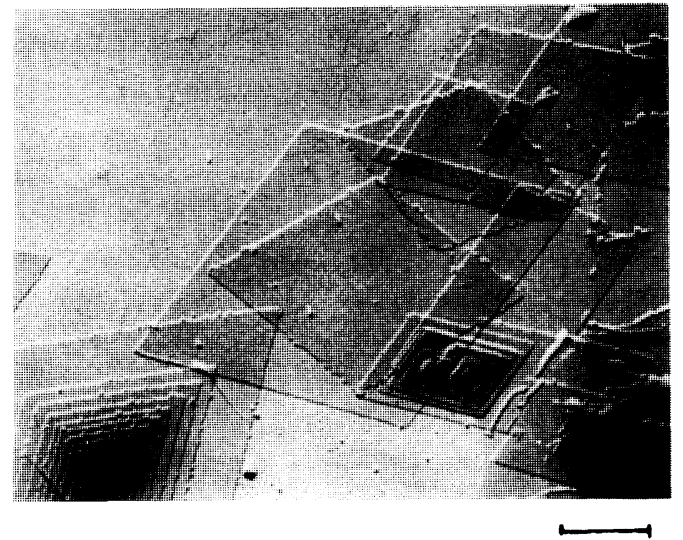

b)

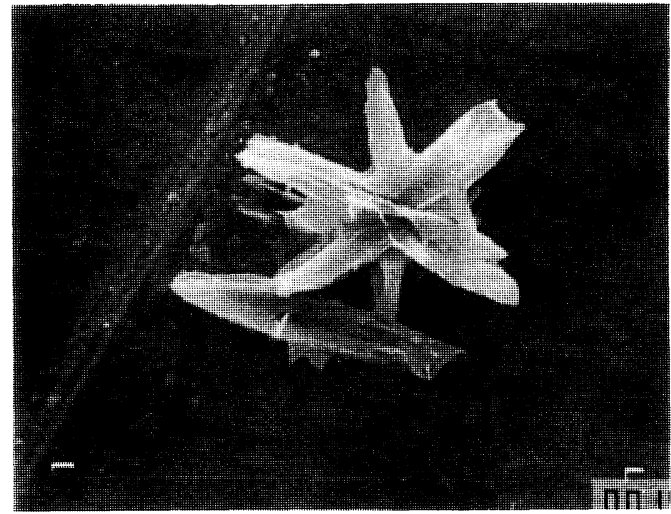

SEM -

d)

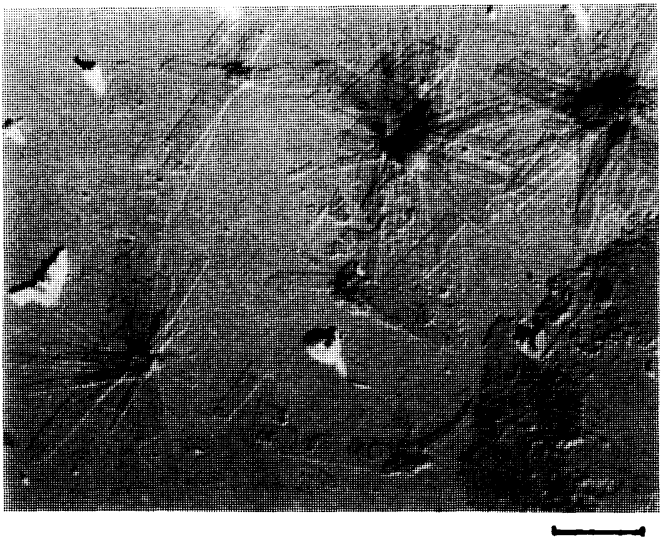

Figure 3. TEM and SEM photographs for the solution-crystallized samples. (a) C160 (PX); (b) C160 (IAAC); (c) C120 (PX); (d) C120 (IAAC) bar indicate $2000 \mathrm{~nm}$.
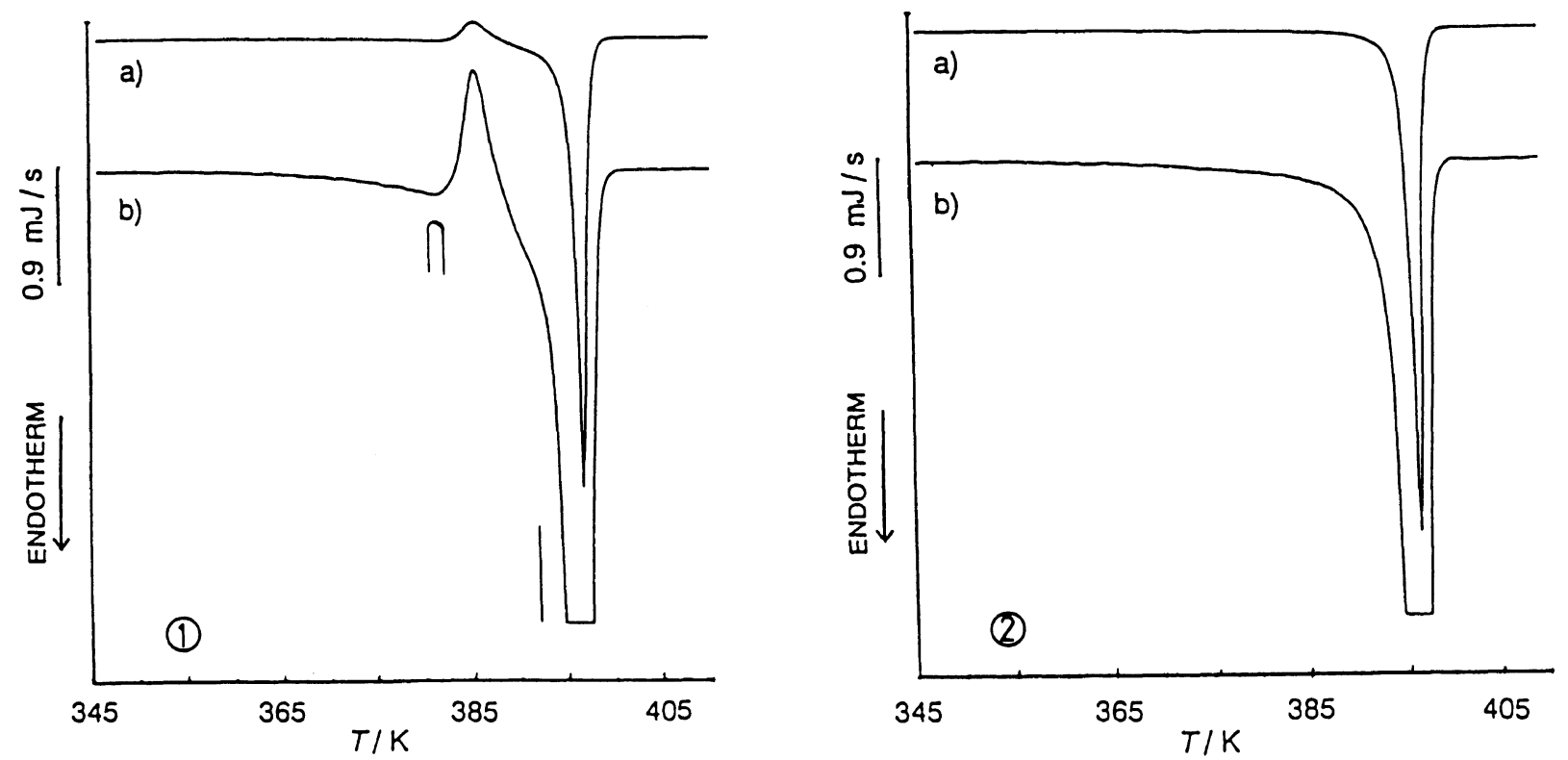

Figure 4. DSC thermograms for solution-crystallized samples of $\mathrm{Cl} 60$ heating rate $3 \mathrm{~K} \mathrm{~min}^{-1}$. Crystallization-solvents: (1) PX; (2) IAAC. (a) normal drawing; (b) enlarged drawing $(\times 25)$.

\section{Polymorphism of Melting Process for Once-Folded Crystals of C160 and C120}

Figure 4 shows DSC thermograms of the $\mathrm{C} 160$ crystals from PX (1) by quenching and from IAAC (2) solutions. Enlarged curves (b) are also shown for distinction of detailed thermal features, taking into account results of TEM observation. The peak at lower tempera- ture side in Figure 4(1) should indicate the melting of folded chain crystal, and the following exothermic peak, the crystallization of extended chain form, which melted at a somewhat higher temperature. Ungar et $a l .{ }^{13}$ reported similar results in the case of bulk crystals of $n-\mathrm{C}_{294} \mathrm{H}_{590}$. Figure 4(2) is the DSC thermogram for the crystal prepared from isothermal crystallization 

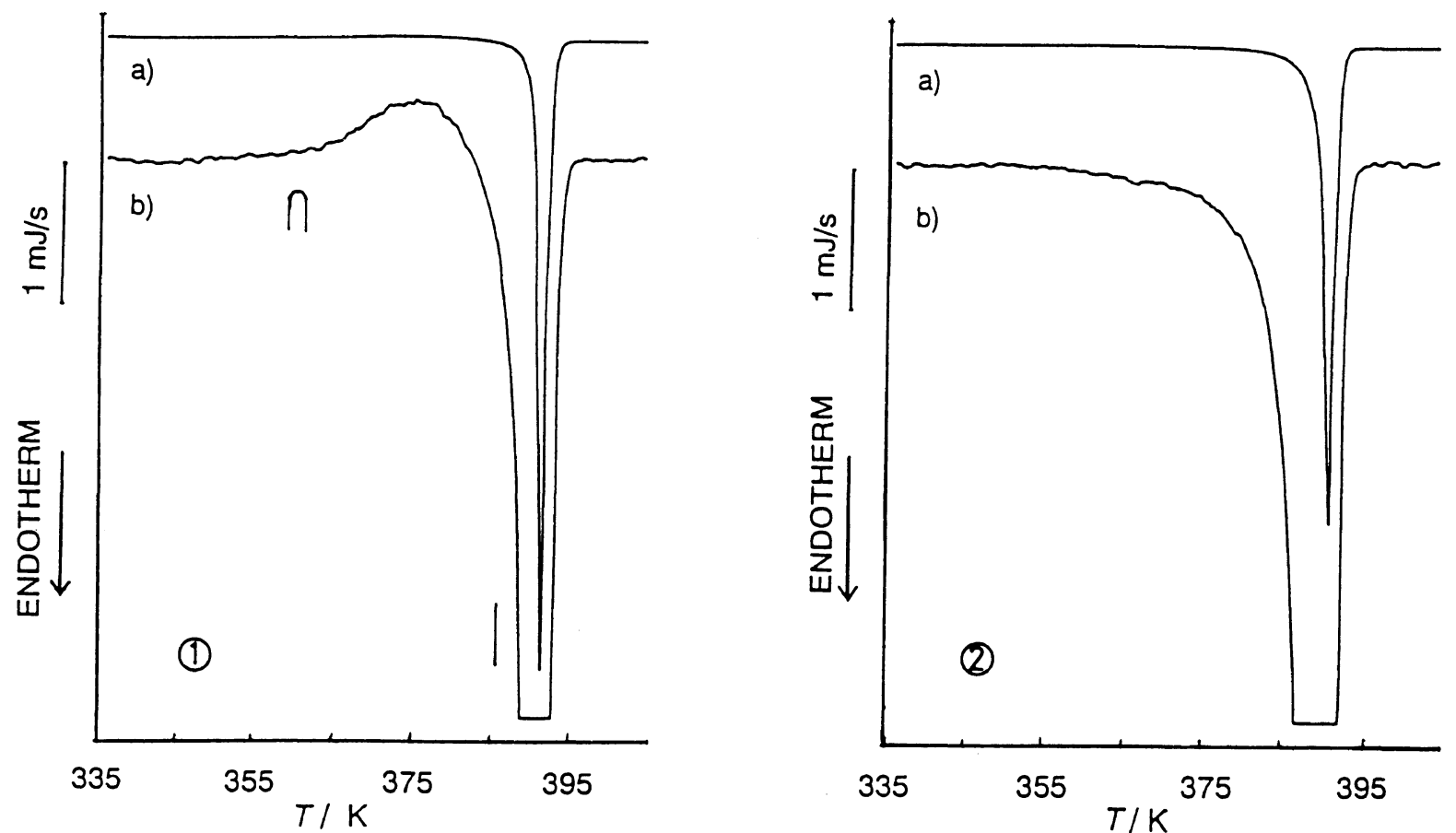

Figure 5. DSC thermograms for solution-crystallized samples of $\mathrm{C} 120$ heating rate $3 \mathrm{~K} \mathrm{~min}^{-1}$. Crystallization-solvents; (1) PX; (2) IAAC. (a) normal drawing; (b) enlarged drawing $(\times 25)$.

at the IAAC solution at $92^{\circ} \mathrm{C}$ for 216 hours, where the enlarged curve also indicates a single endothermic peak at $394.5 \mathrm{~K}$. This $T_{\mathrm{m}}$ is higher by $2.3 \mathrm{~K}$ than that of the previous synthetic product ${ }^{6}$ and proves the effects of purification of starting material. The growth of extended chain crystals is confirmed. Figure 5 shows DSC thermograms for the $\mathrm{C} 120$ crystals when the sample was subjected to crystallization at the conditions as in the case of $\mathrm{C} 160$. The thermal behavior for C120 crystals was similar to that of $\mathrm{C} 160$; i.e., the formation of oncefolded chain crystal was confirmed to occur in the case of $\mathrm{C} 120$. The heating rate was $3 \mathrm{~K} \mathrm{~min}^{-1}$ as the case of C160 crystal. With the standard heating rate of 0.5 $\mathrm{K} \min ^{-1}$, the endothermic peak of once-folded chain crystal became ambiguous, which suggests that stabilization from a metastable state of once-folded chain crystal to the extended chain crystal gradually occurs at such a slow heating rate. From this point of view, cross-linking experiment ${ }^{14}$ for amorphous parts was made by $\gamma$-ray irradiation for the purpose of inhibiting melt-recrystallization. However, this experiment was unsuccessful for $\mathrm{C} 160$ and $\mathrm{C} 120$ crystals due to the absence of a double bonds in the parts to be crosslinked. The $\mathrm{C} 120$ crystals from IAAC solution had an endothermic peak temperature of $392.4 \mathrm{~K}$, which agrees with $392.7 \mathrm{~K}$ calculated from the Broadhurst's equation ${ }^{9}$ as in the case of C160 from IAAC solution.

\section{Effects of Heat Treatment for Once-Folded Chain Crys- tals}

Estimation of the melting point of once-folded chain crystals was made by heat treatment method since $\gamma$-ray irradiation was not applicable as mentioned above. Figure 6 shows DSC thermograms for heat treated C160 crystals and the $\mathrm{C} 160$ crystal as grown. The endothermic peak, indicated by the arrow, of the once-folded chain crystals became sharp and peak temperature became

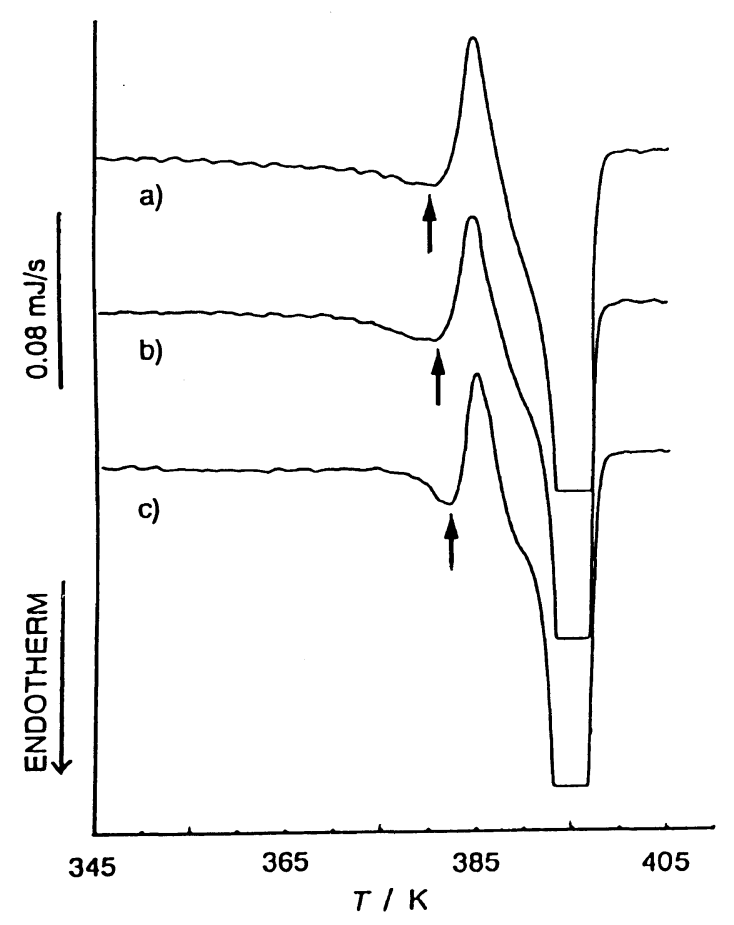

Figure 6. DSC thermograms for annealed samples of the solutioncrystallized $\mathrm{C} 160$. Heating rate $3 \mathrm{~K} \mathrm{~min}^{-1}$. (a) as grown sample compared with (b),(c); (b) $T_{\mathrm{a}} 94^{\circ} \mathrm{C}, 30 \mathrm{~min}$. (c) $T_{\mathrm{a}} 104^{\circ} \mathrm{C}, 30 \mathrm{~min}$.

higher with annealing time. The once-folded chain crystal here is considered "as grown" type shown schematically in Figure 7 which is a metastable state containing various defects during crystallization process indicated by arrows. Highly stable crystals like $\mathbf{C 1}$ can be measured directly for the crystal from IAAC as shown in Figure 4(2) and Figure 5(2). In less stable crystals of $\mathbf{C} 2, \mathbf{C} 3$, and $\mathbf{C} 4$ which have Gibbs's free energy $G$ in the order $G_{\mathrm{C} 4}>G_{\mathrm{C} 3}>G_{\mathrm{C} 2}>G_{\mathrm{C} 1},{ }^{15}$ thermal motion starts at a considerably low temperature during heating and the 


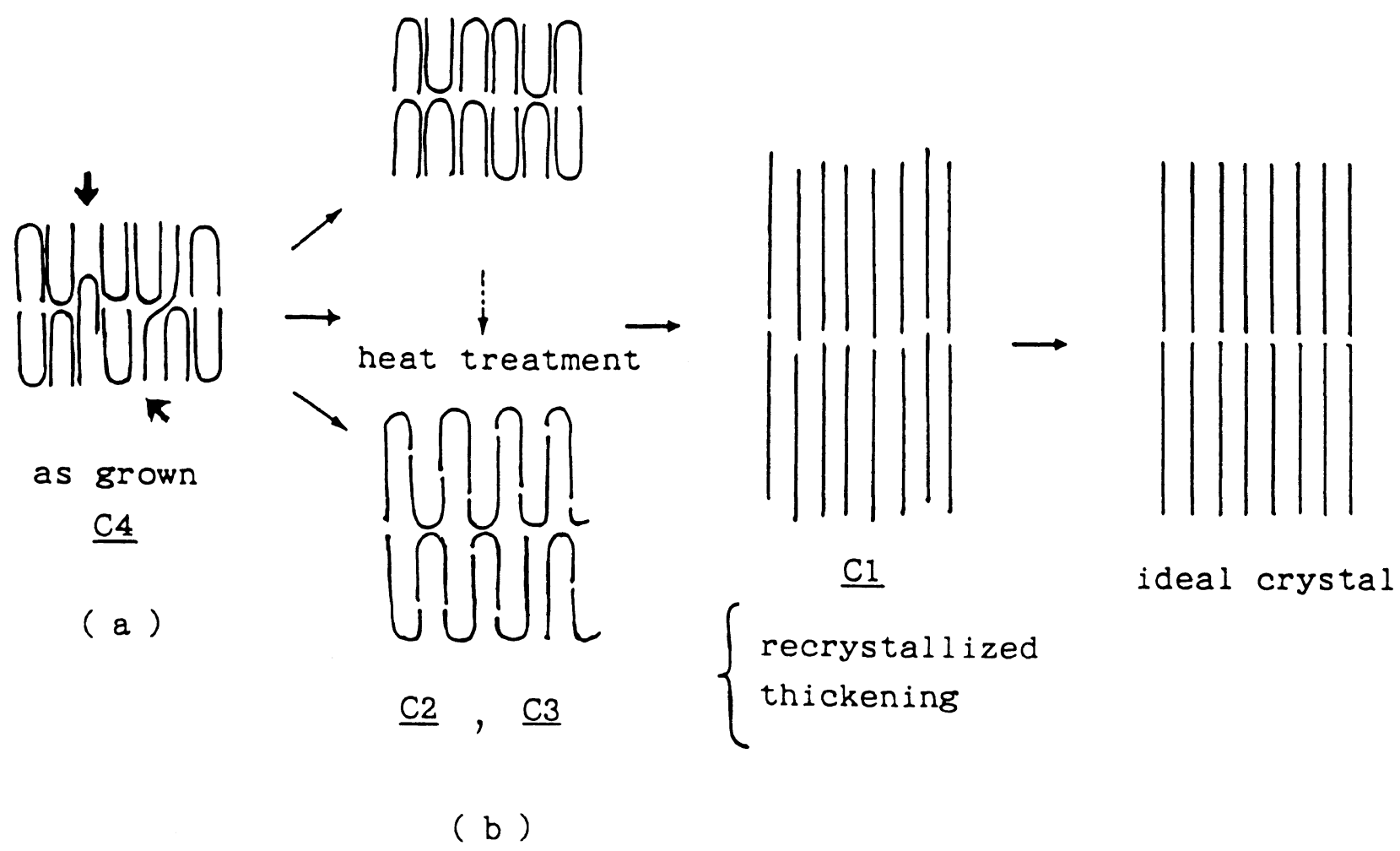

Figure 7. Schematic representation of molecular structure of once-folded chain crystals. (a) as grown type; (b) annealed type.

a)

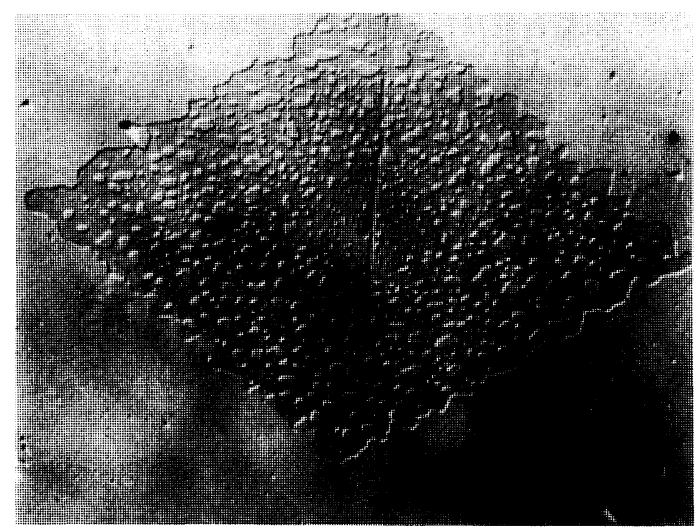

b)

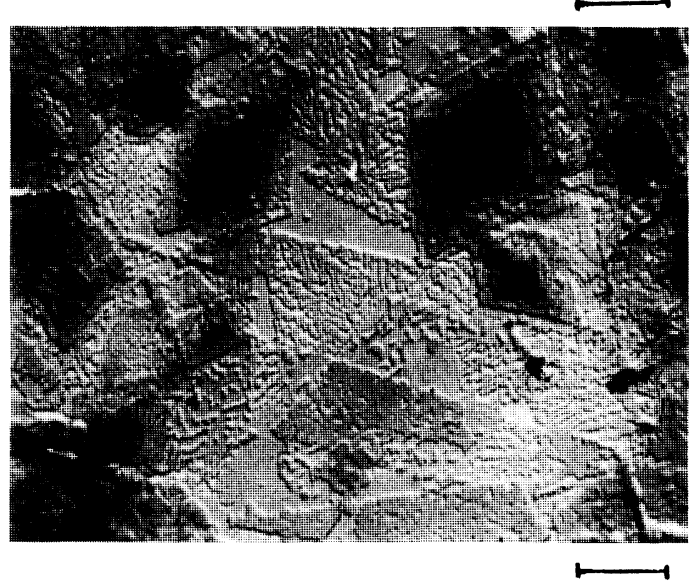

Figure 8. TEM photographs for annealing samples of solutioncrystallized $\mathrm{C} 160$ and $\mathrm{C} 120$. (a) $\mathrm{Cl60}, T_{\mathrm{a}} 94^{\circ} \mathrm{C}, 30 \mathrm{~min}$; (b) $\mathrm{C} 120, T_{\mathrm{a}}$ $84.5^{\circ} \mathrm{C}, 30 \mathrm{~min}$ bar indicate $2000 \mathrm{~nm}$. metastable crystals transform to more stable structure $(\mathrm{C} 4 \rightarrow \mathrm{C} 3, \mathrm{C} 2)$ through the reorganization before melting. The enlarged DSC curve suggests that the rearrangement occurs at considerably low temperature. TEM photographs showing morphology of $\mathrm{C} 160$ and $\mathrm{C} 120$ crystals after heat treatment are shown in Figure 8. The specimens were prepared by hanging a crystal suspension on carbon film on mesh, drying the solvent of suspension enough, and subjecting this sheet mesh to heat treatment in an ample. Holes representing thickening of layer lamella observed by Hirai et al. ${ }^{16}$ in TEM were observed at many sites on the crystal surface. Thickness determined from shadow for $\mathrm{C} 160$ was about $7 \mathrm{~nm}$ larger than folded chain length. This might be ascribed to structural change accompanied by thickening although confirmation by the long period measurement of the heat treated crystal is required. The melting point of recrystallized sample (extended chain crystal) at higher temperature changed little with heat treatment, but the endothermic peak temperature rose, and enthalpy of fusion increased. It is quite possible that thickening of layer lamella occurs and subsequent recrystallization after melting, and remelting follows. Hence, determination of the melting point of once-folded chain crystal is considered to require change of structure (highly stable state) by heat treatment at a substantially low temperature. Discussion is possible without regarding partial melting or other matters frequently observed for a poly-disperse sample because of the high purity in this study.

\section{CONCLUSIONS}

$\mathrm{C} 80, \mathrm{C} 102, \mathrm{C} 120$, and $\mathrm{C} 160$ of high purity were synthesized by Wurtz condensation. For C120 and C160, 
once-folded chain crystals were formed by solution crystallization. Folding of $n$-alkane molecular chain was found to take place from C120. The thickness of layer lamella was on an average, $7.8 \mathrm{~nm}$ for C120 and $10.0 \mathrm{~nm}$ for $\mathrm{C} 160$, or half the extended chain length. In the DSC thermograms of $\mathrm{C} 160$ crystals, an exothermic peak indicates recrystallization to extend crystal chain length following melting of once-folded chain crystals, which melted at some what higher temperature.

Acknowledgment. This work was supported in part by Gran-in-Aid for Development Scientific Research (No. 03555191) from the Ministry of Education, Science, and Culture of Japan. We are grateful to Prof. N. Nemoto for discussion and helpful comments during preparation of this manuscript.

\section{REFERENCES}

1. A. Keller, Phil. Mag., 2, 1171 (1957); W. W. Fischer, Z. Naturforsch, 129, 753 (1957); D. H. Till, Jr., J. Polym. Sci., 24, 301 (1957).
2. K. S. Lee and G. Wegner, Makromol. Sci. Rapid Commun., 6, 203 (1985).

3. L. Bidd and M. C. Whiting, Chem. Commun., 543 (1985).

4. K. Takamizawa, Netsusokutei, 16, 112 (1989); K. Takamizawa, Y. Urabe, and M. Hashiguti, Polym. Prep. Jpn., 21, 571 (1972).

5. W. H. Carothers, J. W. Hill, J. W. Kibby, and R. A. Jacobson, J. Am. Chem. Soc., 52, 527 (1930); W. Heitz, Th. Wirth, R. Peters, G. Strobl, and E. W. Fischer, Makromol. Chem., 162, 63 (1972).

6. K. Takamizawa, Y. Sasaki, K. Kono, and Y. Urabe, Rep. Progr. Polym. Phys. Jpn., 19, 285 (1976).

7. K. Takamizawa, T. Sonoda, and Y. Urabe, Engineering Science Reports Kyushu University, 10, 363 (1989).

8. Y. Urabe and K. Takamizawa, Polym. J., 26, 283 (1994).

9. M. G. Broadhurst, J. Res. Natl. Bur. Stand., 66A, 241 (1962).

10. K. Takamizawa, Y. Urabe, and T. Hara, Rep. Progr. Polym. Phys. Jpn., 12, 179 (1969).

11. A. Toda, Faraday Discuss., 95, 127 (1993).

12. F. C. Frank, J. Cryst. Growth, 22, 233 (1974).

13. G. Ungar, J. Stejny, A. Keller, and I. Bidd, M. C. Whiting, Science, 229, 386 (1985).

14. K. Takamizawa, Y. Fukahori, and Y. Urabe, Makromol. Chem., 128, 236 (1969)

15. K. Takamizawa, "Series of Functional Polymer Materials," Vol. 3, Kyoritsu Publishing Co., Tokyo, 1993, p 296.

16. N. Hirai, T. Mitsuhata, and Y. Yamasita, Kobunshi Kagaku, 189, 33 (1961). 